\title{
Design of Pulsewidth-Modulation Based Sliding Mode Controllers for Power Converters in Discontinuous Conduction Mode
}

\author{
Siew-Chong Tan*, Y. M. Lai*, Chi K. Tse*, Luis Martinez-Salamero ${ }^{\dagger}$, and Angel Cid-Pastor ${ }^{\ddagger}$ \\ ${ }^{*}$ Department of Electronic and Information Engineering \\ Hong Kong Polytechnic University, Hong Kong, China \\ E-mail: ensctan@polyu.edu.hk; enymlai@inet.polyu.edu.hk; encktse@polyu.edu.hk \\ ${ }^{\dagger}$ Department of Electrical, Electronic and Automatic Control Engineering \\ Rovira i Virgili University, Taragona, Spain. \\ E-mail: luis.martinez@urv.net \\ ‡LAAS-CNRS , 7, Avenue du Colonel Roche, 31077 Toulouse Cedex 4, France. \\ E-mail: acid@laas.fr
}

\begin{abstract}
This paper presents an approach of designing fixed-frequency pulsewidth-modulation based sliding mode controllers for power converters operating in the discontinuous conduction mode. The basic concept and design issues are discussed. Preliminary verification and evaluation of the derived controllers are performed through computer simulations using precise models of the systems.
\end{abstract}

\section{INTRODUCTION}

Power converters that operate in the discontinuous conduction mode (DCM) enjoys a faster transient response (due to its low inductance) at the expense of higher device stresses. Nevertheless, it is still a popular operating mode for low power applications. The typical controller used in these converters are the conventional PWM current or voltage controllers. Designed from the small-signal models of the converters, these controllers are optimal only in regions within the specified operating condition. They give poor performance in operating condition much deviated from the specified point. Hence, to truly benefit from the advantages of employing converters operating in DCM without sacrificing the regulation property over a wide range of operating condition, it is necessary to search for better means of controlling these converters. One such alternative is to adopt the nonlinear controller: sliding mode controller.

The sliding mode (SM) controller is well-known for its robustness, stability, and good regulation properties over wide operating conditions. It is deemed to be a better candidate in converters' application than other nonlinear controllers for its relative ease of implementation [1]-[3]. In particular, the fixed-frequency pulsewidth modulation (PWM) based SM controllers, which are basically pulsewidth modulators that employ control signals derived from SM control technique, are found to be more suited for practical implementation in power converters [1], [4]-[7]. However, the results presented in these papers are only valid for converters in continuous conduction mode (CCM) operation. They are not applicable to converters operating in DCM. There is also no report on how such controllers can be derived.

In view of this, this paper introduces an approach that can be used for deriving fixed-frequency pulsewidth-modulation based sliding mode controllers for power converters operating in DCM. The objective of the paper is to communicate the basic concept, mathematical derivation, and design issues of such controllers. Preliminary verification and evaluation of these controllers are performed through computer simulations using precise models of the systems.

\section{The Proposed Design Approach}

SM controllers for DCM converters adopt the same control philosophy, and therefore, the same control architecture as the SM controllers for CCM converters. The difference lies in the converter's mathematical model adopted in the controller design.

\section{A. Developing DCM Converter's State-Space Model}

Due to its additional zero-inductor-current stage, the statespace model of the DCM converter for SM controller design is more complicated than that of the CCM converter. It is necessary to consider all three stages of the DCM operation when constructing the model. For this purpose, the DCM converter models is developed by introducing additional terms, known as the virtual switching components, into the model. Such analogy is only theoretical. No physical switch is added to the converter.

For example, in the case of the buck, boost, and buckboost converters, the virtual switching components $u_{\mathrm{L}}=$ $\left\{\begin{array}{ll}1 & \text { when } i_{L}>0 \\ 0 & \text { when } i_{L}=0\end{array}\right.$ and $u_{\mathrm{B}}=\left\{\begin{array}{ll}1 & \text { when } i_{L}>0 \text { and } u=0 \\ 0 & \text { when } i_{L}=0 \text { and } u=0\end{array}\right.$, on top of the actual physical switching component $u$, can be introduced into the models. Here, logic 1 implies the "ON" 
state and logic 0 implies the "OFF" state of the respective switching components $u, u_{\mathrm{L}}$, and $u_{\mathrm{B}}$.

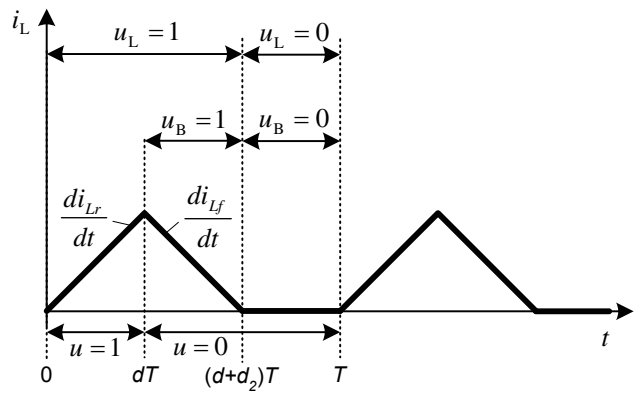

Fig. 1. Inductor current behavior of a DC-DC converter in DCM operation.

TABLE I

State-Space Model of The Various Converters in DCM

\begin{tabular}{|c|c|c|c|c|}
\hline Converter & $\frac{d i_{L r}}{d t}$ & $\frac{d i_{L f}}{d t}$ & $i_{L}$ & $v_{\mathrm{O}}$ \\
\hline Buck & $\frac{v_{\mathrm{i}}-v_{\mathrm{O}}}{L}$ & $-\frac{v_{\mathrm{O}}}{L}$ & $\frac{\int\left[v_{\mathrm{i}} u-v_{\mathrm{o}} u_{\mathrm{L}}\right] d t}{L}$ & $\frac{\int\left[i_{L}-i_{r}\right] d t}{C}$ \\
\hline Boost & $\frac{v_{\mathrm{i}}}{L}$ & $\frac{v_{\mathrm{i}}-v_{\mathrm{O}}}{L}$ & $\frac{\int\left[v_{\mathrm{i}} u+\left(v_{\mathrm{i}}-v_{\mathrm{O}}\right) u_{\mathrm{B}}\right] d t}{L}$ & $\frac{\int\left[i_{L} \bar{u}-i_{r}\right] d t}{C}$ \\
\hline Buck-boost & $\frac{v_{\mathrm{i}}}{L}$ & $-\frac{v_{\mathrm{O}}}{L}$ & $\frac{\int\left[v_{\mathrm{i}} u-v_{\mathrm{O}} u_{\mathrm{B}}\right] d t}{L}$ & $\frac{\int\left[i_{L} \bar{u}-i_{r}\right] d t}{C}$ \\
\hline
\end{tabular}

Fig. 1 illustrates the typical inductor current behavior of these converters in DCM operation. The respective rate of change of inductor currents (i.e. $\frac{d i_{L r}}{d t}$ and $\frac{d i_{L f}}{d t}$ ) and statespace inductor current $i_{L}$ descriptions are stated in Table I. The relationship between the converters' state-space output voltage $v_{\mathrm{o}}$, inductor current $i_{L}$, switching signal $u / \bar{u}$, and load current $i_{r}$ are also illustrated. This represents the sort of the state-space model required for deriving the PWM based SM controller for DCM converters.

\section{B. Modeling The System In Terms of Its Control Variable}

The method of modeling the controller/converter system is the same for both cases of CCM and DCM converters. It requires only the substitution of the converter's state-space model into the control equations.

Assume that we are modeling a PID sliding mode voltage controlled (SMVC) DC-DC converter as shown in Fig. 2. Here $C, L$, and $r_{\mathrm{L}}$ denote the capacitance, inductance, and instantaneous load resistance of the converters respectively; $i_{C}, i_{L}$, and $i_{r}$ denote the instantaneous capacitor, inductor, and load currents respectively; $V_{\text {ref }}, v_{\mathrm{i}}$, and $v_{\mathrm{o}}$ denote the reference, instantaneous input, and instantaneous output voltages respectively; $\beta$ denotes the feedback network ratio; and $u=0$ or 1 is the switching state of power switch $S_{\mathrm{W}}$ and $\bar{u}=1-u$ is the inverse logic of $u$.

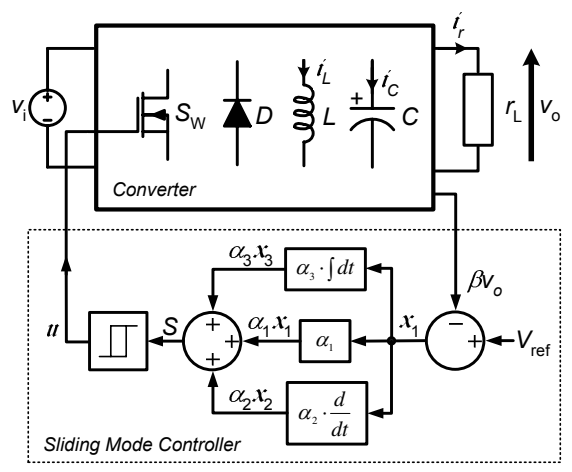

Fig. 2. General form of a PID SMVC converter.

The SM control law adopts a switching function

$$
u= \begin{cases}1 & \text { when } S>0 \\ 0 & \text { when } S<0\end{cases}
$$

where the instantaneous state variable's trajectory is

$$
S=\alpha_{1} x_{1}+\alpha_{2} x_{2}+\alpha_{3} x_{3}=\boldsymbol{J}^{\mathrm{T}} \boldsymbol{x}
$$

with $\boldsymbol{J}^{\mathrm{T}}=\left[\begin{array}{lll}\alpha_{1} & \alpha_{2} & \alpha_{3}\end{array}\right]$ and $\alpha_{1}, \alpha_{2}$, and $\alpha_{3}$ representing the control parameters termed as sliding coefficients; and the control variables $\boldsymbol{x}$ expressed as

$$
\boldsymbol{x}=\left[\begin{array}{l}
x_{1} \\
x_{2} \\
x_{3}
\end{array}\right]=\left[\begin{array}{c}
V_{\mathrm{ref}}-\beta v_{\mathrm{o}} \\
\frac{d\left(V_{\mathrm{ref}}-\beta v_{\mathrm{o}}\right)}{d t} \\
\int\left(V_{\mathrm{ref}}-\beta v_{\mathrm{o}}\right) d t
\end{array}\right]
$$

where $x_{1}, x_{2}$, and $x_{3}$ represents the voltage error, the voltage error dynamics (or the rate of change of voltage error), and the integral of voltage error, respectively [6]. Quite simply, the substitution of the converters' behavioral models in Table I into (3) followed by a time derivative produces the statespace descriptions required for deriving the PWM based SM controller. The descriptions of the buck, boost, and buck-boost converters are summarized in Table II in the standard form

$$
\dot{\boldsymbol{x}}=\mathbf{A} \boldsymbol{x}+\mathbf{B} v+\mathbf{D}
$$

where $v=u$ or $\bar{u}$ (depending on topology).

\section{Deriving the Controller in PWM Form}

Equations (1) and (2) describe the control equations needed for implementing the SM controller in conventional HM form. For implementation in the PWM form, the control equations must be translated. This is possible by firstly obtaining its equivalent control function, which is then mapped onto the duty cycle function of the pulse-width modulator [7]. The approach is similar to that adopted for deriving PWM based controllers for CCM converters. However, an additional step of mathematical approximation which uses the average models of the DCM converters to estimate the equivalent virtual component is required in the process of this translation. Illustration is provided for the buck converter. 
TABLE II

MATRIX DESCRIPTIONS OF THE SMVC CONVERTERS IN DCM

\begin{tabular}{|c|c|c|c|c|}
\hline Converter & $\mathbf{A}$ & $\mathbf{B}$ & $\mathbf{D}$ & $v$ \\
\hline Buck & {$\left[\begin{array}{ccc}0 & 1 & 0 \\
0 & -\frac{1}{r_{\mathrm{L} C}} & 0 \\
1 & 0 & 0\end{array}\right]$} & {$\left[\begin{array}{c}0 \\
-\frac{\beta v_{\mathrm{i}}}{L C} \\
0\end{array}\right]$} & {$\left[\begin{array}{c}0 \\
\frac{\beta v_{\mathrm{O}}}{L C} u_{\mathrm{L}} \\
0\end{array}\right]$} & $u$ \\
\hline Boost & {$\left[\begin{array}{ccc}0 & 1 & 0 \\
0 & -\frac{1}{r_{\mathrm{L} C}} & 0 \\
1 & 0 & 0\end{array}\right]$} & {$\left[\begin{array}{c}0 \\
\frac{\beta v_{\mathrm{O}}}{L C} u_{\mathrm{B}}-\frac{\beta v_{\mathrm{i}}}{L C} u_{\mathrm{B}} \\
0\end{array}\right]$} & {$\left[\begin{array}{l}0 \\
0 \\
0\end{array}\right]$} & $\bar{u}$ \\
\hline Buck-boost & {$\left[\begin{array}{ccc}0 & 1 & 0 \\
0 & -\frac{1}{r_{\mathrm{L} C}} & 0 \\
1 & 0 & 0\end{array}\right]$} & {$\left[\begin{array}{c}0 \\
\frac{\beta v_{\mathrm{O}}}{L C} u_{\mathrm{B}} \\
0\end{array}\right]$} & {$\left[\begin{array}{l}0 \\
0 \\
0\end{array}\right]$} & $\bar{u}$ \\
\hline
\end{tabular}

\section{Example: Buck converter}

Equating $\frac{d S}{d t}=\boldsymbol{J}^{\mathrm{T}} \mathbf{A} \boldsymbol{x}+\boldsymbol{J}^{\mathrm{T}} \mathbf{B} u_{\text {eq }}+\boldsymbol{J}^{\mathrm{T}} \mathbf{D}=0$ yields the equivalent control function

$$
\begin{array}{r}
u_{\mathrm{eq}}=-\frac{\beta L}{\beta v_{\mathrm{i}}}\left(\frac{\alpha_{1}}{\alpha_{2}}-\frac{1}{r_{\mathrm{L}} C}\right) i_{C}+\frac{\alpha_{3} L C}{\alpha_{2} \beta v_{\mathrm{i}}}( \\
\left.V_{\mathrm{ref}}-\beta v_{\mathrm{o}}\right) \\
+\frac{v_{\mathrm{o}}}{v_{\mathrm{i}}} u_{\text {Leq }}
\end{array}
$$

where both $u_{\text {eq }}$ and $u_{\text {Leq }}$ are continuous and bounded by 0 and 1. Specifically, $u_{\mathrm{eq}}$ is a smooth function of the discrete input function $u$, and $u_{\text {Leq }}$ is the resulted equivalent component of the discrete virtual switching component $u_{\mathrm{L}}$.

Then, by substituting (5) into $0<u_{\text {eq }}<1$ and multiplying by $\beta v_{\mathrm{i}}$, the function can be expressed as

$$
\begin{array}{r}
0<-\beta L\left(\frac{\alpha_{1}}{\alpha_{2}}-\frac{1}{r_{\mathrm{L}} C}\right) i_{C}+L C \\
\frac{\alpha_{3}}{\alpha_{2}}\left(V_{\text {ref }}-\beta v_{\mathrm{o}}\right) \\
+\beta v_{\mathrm{o}} u_{\text {Leq }}<\beta v_{\mathrm{i}} .
\end{array}
$$

Next, $u_{\text {Leq }}$ can be approximated from well-established mathematical models of the buck converter. Firstly, for the buck converter in DCM, the duty cycle is described as

$$
D_{\mathrm{DCM}}=\sqrt{\frac{v_{\mathrm{o}}^{2}}{v_{\mathrm{i}}\left(v_{\mathrm{i}}-v_{\mathrm{o}}\right)} \frac{2 L}{r_{\mathrm{L}} T}} .
$$

Additionally, the duty cycle for the turn-off period in DCM operation is

$$
D_{2}=\frac{v_{\mathrm{i}}-v_{\mathrm{o}}}{v_{\mathrm{o}}} D_{\mathrm{DCM}}=\sqrt{\frac{v_{\mathrm{i}}-v_{\mathrm{o}}}{v_{\mathrm{i}}} \frac{2 L}{r_{\mathrm{L}} T}} .
$$

Since the maximum time duration that $u_{L}=1$ can exist is $T$ (during CCM operation), and in DCM operation $u_{L}=1$ is applied for the time duration $\left(D_{\mathrm{DCM}}+D_{2}\right) T$, the equivalent virtual switching component applied to the system in DCM operation can be described as

$$
u_{\mathrm{Leq}}=\frac{\left(D_{\mathrm{DCM}}+D_{2}\right) T}{T}=\sqrt{\frac{v_{\mathrm{i}}}{v_{\mathrm{i}}-v_{\mathrm{o}}} \frac{2 L}{r_{\mathrm{L}} T}} .
$$

Finally, the mapping of (6) onto the duty ratio control $D$, where $0<D=\frac{v_{\mathrm{c}}}{\hat{v}_{\mathrm{ramp}}}<1$, gives the relationships of the control signal $v_{\mathrm{c}}$ and ramp signal $\hat{v}_{\text {ramp }}$ as

$$
\left\{\begin{array}{l}
v_{\mathrm{c}}=-\beta L\left(\frac{\alpha_{1}}{\alpha_{2}}-\frac{1}{r_{\mathrm{L}} C}\right) i_{C} \\
+\frac{\alpha_{3}}{\alpha_{2}} L C\left(V_{\mathrm{ref}}-\beta v_{\mathrm{o}}\right) \\
\quad+\beta v_{\mathrm{o}} u_{\text {Leq }} \\
\hat{v}_{\mathrm{ramp}}=\beta v_{\mathrm{i}}
\end{array}\right.
$$

The controller equations for the boost and the buck-boost converters can be derived using the same approach. Table III shows the simplified control equations required for implementing the respective PWM based SMVC converter operating in DCM. The representation takes into consideration that $r_{\mathrm{L}}=$ $\frac{v_{0}}{i_{r}}$.

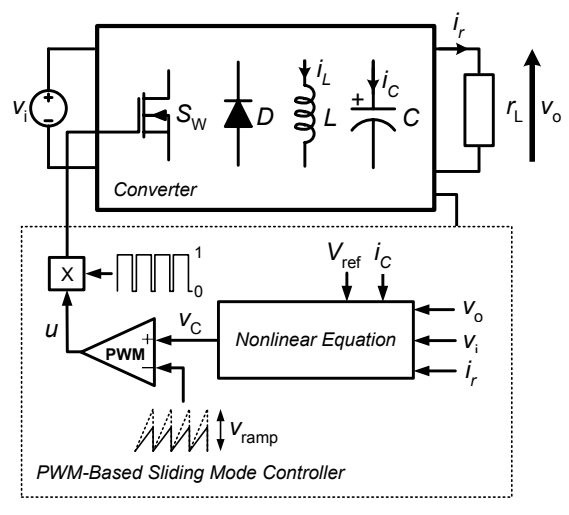

Fig. 3. General form of a PID PWM based SMVC converter for DCM operation.

Fig. 3 shows a general form of the derived PWM based SMVC converters for DCM operation. Notably, the derived controllers are highly nonlinear, requiring various square root, division, multiplier, addition, and subtraction operators in their computations. It accurately represents the sort of nonlinear controllers truly required for providing good control to DCM converters operating over a wide variation of input/load range.

\section{SLIDing Mode Conditions}

The implementation of the proposed controllers in Fig. 3 does not automatically qualify them as SM controllers. Three necessary conditions: the hitting condition, existence condition, and stability condition, must be complied. Hitting condition has been satisfied by the control law (1) [9]. This section discusses the compliance of the remaining conditions.

\section{A. Compliance to Existence Conditions}

The method of ensuring the existence of SM operation for the DCM converter system is basically similar to the case of the CCM converter system, despite the structural difference in the composition of their state variable trajectory $S$. Fig. 4 illustrates the difference between the state variable trajectory behavior in CCM and DCM operations.

Typically, for SM controlled CCM converters, all (both) the structures of the state variable trajectory must comply the local reachability condition $\lim _{S \rightarrow 0} S \cdot \frac{d S}{d t}<0$ under their 
TABLE III

Control Equations of PWM BASED SMVC CONVERTERs In DCM

\begin{tabular}{|c|c|c|}
\hline Converter & $v_{\mathrm{c}}$ & $\hat{v}_{\text {ramp }}$ \\
\hline Buck & $-K_{\mathrm{p} 1} i_{C}+K_{\mathrm{p} 2}\left(V_{\mathrm{ref}}-\beta v_{\mathrm{o}}\right)+K_{\mathrm{p} 3} \sqrt{\frac{v_{\mathrm{i}} v_{\mathrm{o}} i_{r}}{v_{\mathrm{i}}-v_{\mathrm{o}}}}$ & $\beta v_{\mathrm{i}}$ \\
\hline Boost & $-K_{\mathrm{p} 1} i_{C}+K_{\mathrm{p} 2}\left(V_{\mathrm{ref}}-\beta v_{\mathrm{o}}\right)+K_{\mathrm{p} 3} \frac{v_{\mathrm{o}}}{v_{\mathrm{i}}} \sqrt{i_{r}\left(v_{\mathrm{o}}-v_{\mathrm{i}}\right)}$ & $K_{\mathrm{p} 3} \frac{v_{\mathrm{o}}}{v_{\mathrm{i}}} \sqrt{i_{r}\left(v_{\mathrm{o}}-v_{\mathrm{i}}\right)}$ \\
\hline Buck-boost & $-K_{\mathrm{p} 1} i_{C}+K_{\mathrm{p} 2}\left(V_{\mathrm{ref}}-\beta v_{\mathrm{o}}\right)+K_{\mathrm{p} 3}\left(1+\frac{v_{\mathrm{o}}}{v_{\mathrm{i}}}\right) \sqrt{v_{\mathrm{o}} i_{r}}$ & $K_{\mathrm{p} 3}\left(1+\frac{v_{\mathrm{o}}}{v_{\mathrm{i}}}\right) \sqrt{v_{\mathrm{o}} i_{r}}$ \\
\hline
\end{tabular}

(a) In CCM operation

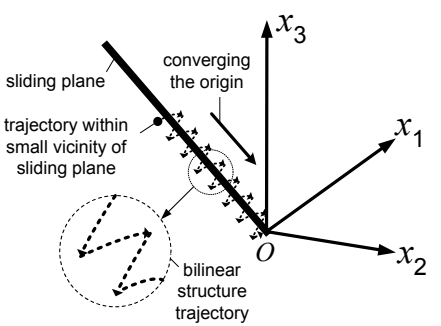

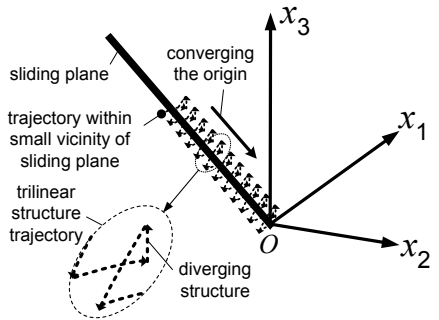

(b) In DCM operation
Fig. 4. Graphical representations of state variables' trajectory's behavior in SM control process: (a) Trajectory behavior in CCM operation - illustrating how bilinear stucture trajectory are maintained within a small vicinity from the sliding plane; and (b) Trajectory behavior in CCM operation - illustrating how tilinear stucture trajectory are maintained within a small vicinity from the sliding plane, even though one of its structure is not directing the trajectory towards the sliding plane.

respective configuration to ensure that the trajectory is moving within a small vicinity from the sliding plane towards the equilibrium, i.e., complying the existence condition. However, for DCM converters which have a trilinear structure trajectory, the abidance of the local reachability condition by the first two structures of the trajectory provides a sufficient condition for the compliance of the existence condition. It is unnecessary for the third structure to also meet the local reachability condition. This is graphically illustrated in Fig. 5. Here, it is shown that as long as Structure 1 and Structure 2 meet the local reachability condition, the direction in which Structure 3 of the state trajectory travels will not affect the overall existence of the SM operation, whereby the trajectory is maintained within the vicinity of the sliding plane and is moving towards the equilibrium.

Intuitively, the local reachability conditions of Structure 1 and Structure 2 in the DCM converter systems is identical to the local reachability conditions derived for both the bilinear structures of the CCM converter systems [7], which in the proposed system, can be expressed as

$$
\left\{\begin{array}{l}
\frac{d S}{d t}_{S \rightarrow 0^{+}}=\boldsymbol{J}^{\mathrm{T}} \mathbf{A} \boldsymbol{x}+\boldsymbol{J}^{\mathrm{T}} \mathbf{B} v_{S \rightarrow 0^{+}}+\boldsymbol{J}^{\mathrm{T}} \mathbf{D}<0 \\
\frac{d S}{d t}_{S \rightarrow 0^{-}}=\boldsymbol{J}^{\mathrm{T}} \mathbf{A} \boldsymbol{x}+\boldsymbol{J}^{\mathrm{T}} \mathbf{B} v_{S \rightarrow 0^{-}}+\boldsymbol{J}^{\mathrm{T}} \mathbf{D}>0
\end{array} .\right.
$$

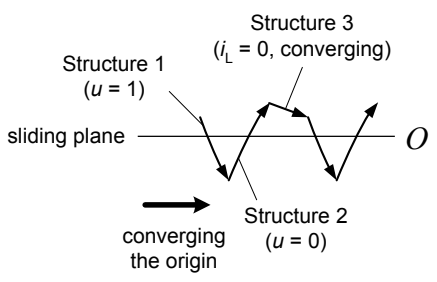

(a) Converging Structure 3

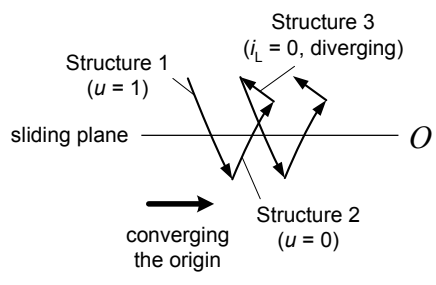

(b) Diverging Structure 3
Fig. 5. Graphical representations of the two possible scenarios of state variables' trajectory's behavior during DCM operation

TABLE IV

EXISTENCE CONDITIONS

\begin{tabular}{|c|}
\hline $0<K_{\mathrm{p} 2}\left(V_{\mathrm{ref}}-\beta v_{\mathrm{o}(\mathrm{SS})}\right)-K_{\mathrm{p} 1} i_{C(\mathrm{SS})}+\beta v_{\mathrm{o}(\mathrm{SS})}<\beta v_{\mathrm{i}(\min )}($ Buck $)$ \\
\hline $0<K_{\mathrm{p} 1} i_{C(\mathrm{SS})}-K_{\mathrm{p} 2}\left(V_{\mathrm{ref}}-\beta v_{\mathrm{o}(\mathrm{SS})}\right)<\beta\left(v_{\mathrm{o}(\mathrm{SS})}-v_{\mathrm{i}(\max )}\right)($ Boost $)$ \\
\hline $0<K_{\mathrm{p} 1} i_{C(\mathrm{SS})}-K_{\mathrm{p} 2}\left(V_{\mathrm{ref}}-\beta v_{\mathrm{o}(\mathrm{SS})}\right)<\beta v_{\mathrm{o}(\mathrm{SS})}($ Buck-boost $)$ \\
\hline
\end{tabular}

Hence, the overall existence conditions of the SMVC buck, boost, and buck-boost converters in DCM operations can be recalled from the previous case of CCM operations [7]. The results are tabulated in Table IV. Here, it is taken into account the complete ranges of operating conditions (minimum and maximum input voltages, i.e., $v_{\mathrm{i}(\min )}$ and $v_{\mathrm{i}(\max )}$, and minimum and maximum load resistances, i.e., $r_{\mathrm{L}(\min )}$ and $\left.r_{\mathrm{L}(\max )}\right)$. Additionally, the state variables $i_{C}$ and $v_{\mathrm{o}}$ are substituted with their expected steady-state parameters, i.e., $i_{C(\mathrm{SS})}$ and $v_{\mathrm{o}(\mathrm{SS})}$, to assure the compliance of the existence condition at least in the small region of the origin [7], [8]. The selection of sliding coefficients must comply the respective inequalities.

Remarks: The direction in which Structure 3 travels will not affect the overall existence of the SM operation. However, if it moves in an opposing direction to the origin, it will significantly prolong the overall settling time of the converters as compared to the CCM converters which have both the 
structures of trajectory moving towards the origin. This is evidenced later in the simulation results.

\section{B. Parameter Selection \& Compliance to Stability Conditions}

The method of assuring the stability of the SMVC DCM converters is the same as the case of CCM converters. For the proposed controllers, the same method of selecting the sliding coefficients based on the desired dynamic properties to arrive at a stable equilibrium as in [6], [7], is adopted.

In the proposed system, the stability condition is easily satisfied by selecting the sliding coefficients for a specific type of dynamical response using one of the following equations:

i) Under-damped response: $\frac{\alpha_{1}}{\alpha_{2}}=\frac{10}{T_{\mathrm{s}}}$ and $\frac{\alpha_{3}}{\alpha_{2}}=\frac{25}{\zeta^{2} T_{\mathrm{s}}^{2}}$, where $T_{\mathrm{s}}$ denotes the settling time and the damping ratio is $\zeta=\sqrt{\frac{\left[\ln \left(\frac{M_{p}}{100}\right)\right]^{2}}{\pi^{2}+\left[\ln \left(\frac{M_{p}}{100}\right)\right]^{2}}}<1$ where $M_{p}$ is the percentage of the peak overshoot.

ii) Critically-damped response: $\frac{\alpha_{1}}{\alpha_{2}}=\frac{10}{T_{\mathrm{s}}}$ and $\frac{\alpha_{3}}{\alpha_{2}}=\frac{25}{T_{\mathrm{s}}{ }^{2}}$.

\section{Simulation Results and Discussions}

The derived PWM based controllers are verified through computer simulation ${ }^{1}$. Results and discussions are provided for the buck and the boost converters.

\section{A. Buck Converter}

TABLE V

SPeCificAtion OF BUCK CONVERTER

\begin{tabular}{|lcc|}
\hline Description & Parameter & Nominal Value \\
\hline Input voltage & $v_{\mathrm{i}}$ & $24 \mathrm{~V}$ \\
Capacitance & $C$ & $150 \mu \mathrm{F}$ \\
Capacitor ESR & $C_{r}$ & $21 \mathrm{~m} \Omega$ \\
Inductance & $L$ & $22 \mu \mathrm{H}$ \\
Inductor resistance & $l_{r}$ & $0.12 \Omega$ \\
Switching frequency & $f_{\mathrm{S}}$ & $200 \mathrm{kHz}$ \\
Minimum load resistance & $r_{\mathrm{L}(\min )}$ & $17.5 \Omega$ \\
Maximum load resistance & $r_{\mathrm{L}(\max )}$ & $120 \Omega$ \\
Desired output voltage & $V_{\mathrm{od}}$ & $12 \mathrm{~V}$ \\
\hline
\end{tabular}

Table $\mathrm{V}$ shows the specification of the DCM buck converter used in the simulation. The PWM based SM voltage controller is designed to give a critically-damped response with a bandwidth of $f_{\mathrm{BW}}=20 \mathrm{kHz}$, i.e., $\tau_{20 \mathrm{kHz}}=7.956 \mu \mathrm{s}$ and $T_{\mathrm{s}(20 \mathrm{kHz})}=39.780 \mu \mathrm{s}$. Designing the controller for maximum load current (i.e. $r_{\mathrm{L}(\mathrm{min})}$ ), and choosing $V_{\text {ref }}=2.5$ $\mathrm{V}$ and $\beta=0.208$, the sliding coefficients are calculated as $\frac{\alpha_{1}}{\alpha_{2}}=251327$ and $\frac{\alpha_{3}}{\alpha_{2}}=15791367040$. Then, the control parameters are determined as $K_{\mathrm{p} 1}=\beta L\left(\frac{\alpha_{1}}{\alpha_{2}}-\frac{1}{r_{\mathrm{L}(\min )} C}\right)=$ $1.15, K_{\mathrm{p} 2}=\frac{\alpha_{3}}{\alpha_{2}} L C=52.1103$, and $K_{\mathrm{p} 3}=\beta \sqrt{\frac{2 L}{T}}=0.6179$.

Fig. 6 shows the steady-state behavior of the SMVC buck converter. Fig. 7(a) shows a plot of the DC output voltage against the different operating load resistances. The results show good load regulation property for the load range of $17.5 \Omega \leq r_{\mathrm{L}} \leq 120 \Omega$ with only a $0.036 \mathrm{~V}$ deviation in $v_{\mathrm{o}}$ (i.e.

\footnotetext{
${ }^{1}$ The simulation is performed using Matlab/Simulink. The step size taken for all simulations is $10 \mathrm{~ns}$.
}
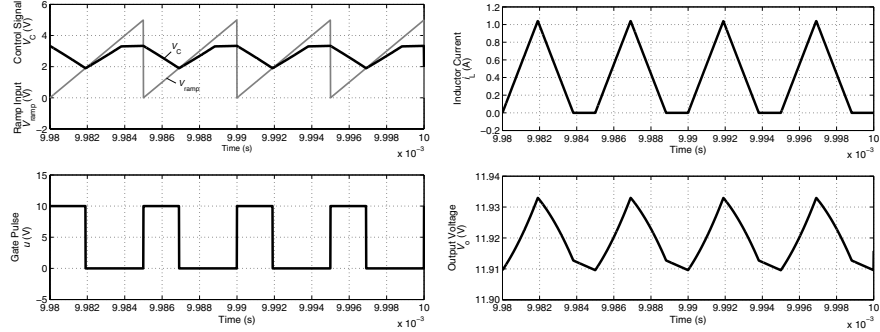

Fig. 6. Waveforms of control signal $v_{\mathrm{c}}$, input ramp $v_{\text {ramp }}$, generated gate pulse $u$ (left), and inductor current $i_{\mathrm{L}}$ and output voltage ripple $v_{\mathrm{O}}$ (right) for the PWM based SMVC buck converter at $v_{\mathrm{i}}=24 \mathrm{~V}$ and $r_{\mathrm{L}}=30 \Omega$.

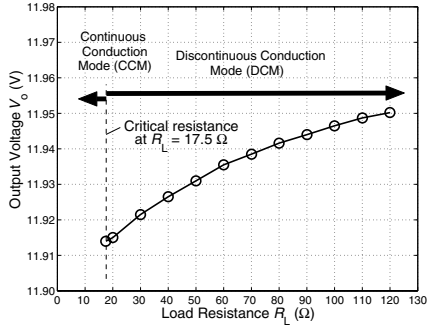

(a) $v_{\mathrm{O}}$ against $r_{\mathrm{L}}$

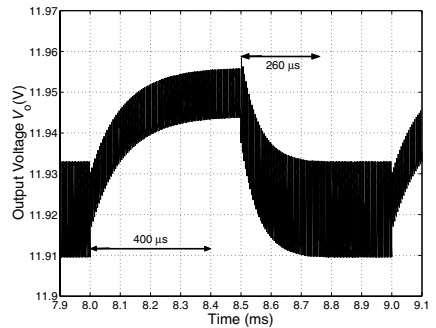

(b) $r_{\mathrm{L}}$ alternating at $30 / 120 \Omega$.
Fig. 7. Static and dynamic performances of the SMVC buck converter.

about $0.3 \%$ of $\left.v_{\mathrm{o}(17.5 \Omega)}\right)$. Fig. 7(b) show the output voltage ripple waveforms of the system at dynamic load condition. The step change responses are critically damped, which conform the designed behavior. The settling times of the system are $400 \mu \mathrm{s}$ and $260 \mu \mathrm{s}$, which are much longer than the ideal SM settling time of around $T_{\mathrm{s}(20 \mathrm{kHz})}=40 \mu \mathrm{s}$. This is due to the opposing direction against the origin in which Structure 3 travels, which significantly prolongs the overall settling time of the converter.

\section{B. Boost Converter}

TABLE VI

SPECIFICATION OF Boost CONVERTER

\begin{tabular}{|lcc|}
\hline Description & Parameter & Nominal Value \\
\hline Input voltage & $v_{\mathrm{i}}$ & $12 \mathrm{~V}$ \\
Capacitance & $C$ & $1000 \mu \mathrm{F}$ \\
Capacitor ESR & $C_{r}$ & $36 \mathrm{~m} \Omega$ \\
Inductance & $L$ & $50 \mu \mathrm{H}$ \\
Inductor resistance & $l_{r}$ & $0.14 \Omega$ \\
Switching frequency & $f_{\mathrm{S}}$ & $200 \mathrm{kHz}$ \\
Minimum load resistance & $r_{\mathrm{L}(\min )}$ & $430 \Omega$ \\
Maximum load resistance & $r_{\mathrm{L}(\max )}$ & $800 \Omega$ \\
Desired output voltage & $V_{\mathrm{od}}$ & $48 \mathrm{~V}$ \\
\hline
\end{tabular}

Table VI shows the specification of the DCM boost converter used in the simulation. The PWM based SM voltage controller is designed to give a critically-damped response with a bandwidth of $f_{\mathrm{BW}}=2 \mathrm{kHz}$, i.e., $\tau_{2 \mathrm{kHz}}=79.56 \mu$ s and $T_{\mathrm{s}(2 \mathrm{kHz})}=397.8 \mu \mathrm{s}$. Designing the controller for maximum load current (i.e. $r_{\mathrm{L}(\min )}$ ), and choosing $V_{\text {ref }}=8 \mathrm{~V}$ and $\beta=$ 0.1667 , the sliding coefficients are calculated as $\frac{\alpha_{1}}{\alpha_{2}}=25132.7$ and $\frac{\alpha_{3}}{\alpha_{2}}=157913670$. Then, the control parameters are determined as $K_{\mathrm{p} 1}=0.209, K_{\mathrm{p} 2}=7.896$, and $K_{\mathrm{p} 3}=0.745$. 

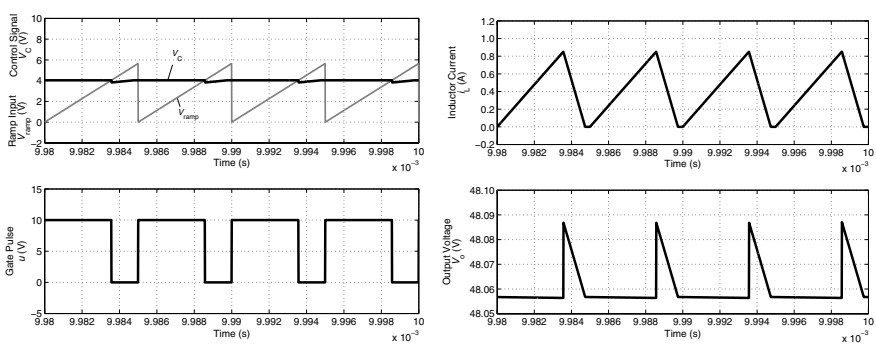

Fig. 8. Waveforms of control signal $v_{\mathrm{c}}$, input ramp $v_{\mathrm{ramp}}$, generated gate pulse $u$ (left), and inductor current $i_{\mathrm{L}}$ and output voltage ripple $v_{\mathrm{O}}$ (right) for the PWM based SMVC boost converter at $v_{\mathrm{i}}=12 \mathrm{~V}$ and $r_{\mathrm{L}}=480 \Omega$.

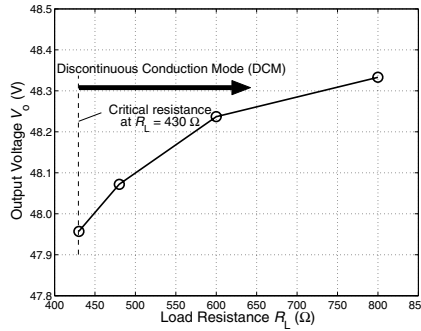

(a) $v_{\mathrm{O}}$ against $r_{\mathrm{L}}$

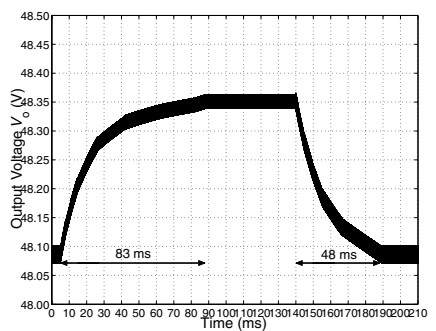

(b) $r_{\mathrm{L}}$ alternating at $480 / 800 \Omega$.
Fig. 9. Static and dynamic performances of the SMVC boost converter.

Fig. 8 shows the steady-state behavior of the SMVC boost converter. Fig. 9(a) shows a plot of the DC output voltage against the different operating load resistances. The results show good load regulation property for the load range of $430 \Omega \leq r_{\mathrm{L}} \leq 800 \Omega$ with only a $0.376 \mathrm{~V}$ deviation in $v_{\mathrm{o}}$ (i.e. about $0.78 \%$ of $\left.v_{\mathrm{o}(430 \Omega)}\right)$. Fig. 9(b) show the output voltage ripple waveforms of the system at dynamic load condition. The step change responses are critically damped, which conform the designed behavior. The settling times of the system are $83 \mathrm{~ms}$ and $48 \mathrm{~ms}$. Again, these settling times are much longer than its ideal SM settling time of around $T_{\mathrm{s}(2 \mathrm{kHz})}=0.4 \mathrm{~ms}$.

\section{Summary of PAPer Contributions}

In this paper, we

i) demonstrate the possibility of constructing state-space models of DCM converters through the use of additional virtual switching components;

ii) propose a method of deriving PWM based SM controllers for DCM converters by approximating the equivalent virtual switching component signal using the average models of the converters;

iii) illustrate that the existence conditions necessary for SM operation to occur in a DCM converter is the same as that in a $\mathrm{CCM}$ converter.

\section{CONCLUSION}

This paper introduces an approach that can be used for deriving fixed-frequency pulsewidth-modulation based sliding mode controllers for power converters operating in discontinuous conduction mode. Using the buck, boost, and buckboost converters as illustrative examples, the basic concept, mathematical derivation, and design issues of such controllers are discussed. Preliminary verification and evaluation of the derived controllers are performed through computer simulations using precise models of the systems. The results show that they are capable of offering good control performances to the respective converters. Potential application of such controllers in practical DCM converters may be considered if existing PWM controllers fails in meeting critical design specification.

\section{REFERENCES}

[1] R. Venkataramanan, A. Sabanoivc, and S. Ćuk, "Sliding mode control of DC-to-DC converters," in Proceedings, IEEE Conference on Industrial Electronics, Control and Instrumentations (IECON), pp. 251-258, 1985.

[2] P. Mattavelli, L. Rossetto, G. Spiazzi, and P. Tenti, "General-purpose sliding-mode controller for $\mathrm{dc} / \mathrm{dc}$ converter applications," in IEEE Power Electronics Specialists Conference Record (PESC), pp. 609-615, June 1993.

[3] E. Alarcon, A. Romero, A. Poveda, S. Porta, and L. Martinez-Salamero "Current-mode analogue integrated circuit for sliding-mode control of switching power converters," Electronic Letters, vol. 38, no. 3, pp. 104106, Jan 2002.

[4] V.M. Nguyen and C.Q. Lee, "Indirect implementations of sliding-mode control law in buck-type converters," in Proceedings, IEEE Applied Power Electronics Conference and Exposition (APEC), vol. 1, pp. 111115, March 1996.

[5] J. Mahdavi, A. Emadi, and H.A. Toliyat, "Application of state space averaging method to sliding mode control of PWM DC/DC converters," in Proceedings, IEEE Conference on Industry Applications (IAS), vol. 2, pp. 820-827, Oct. 1997.

[6] S.C. Tan, Y.M. Lai, C.K. Tse, and M.K.H. Cheung, "A fixed-frequency pulse-width-modulation based quasi-sliding mode controller for buck converters", IEEE Transactions on Power Electronics, vol. 20, no. 6, pp. 1379-1392, Nov. 2005.

[7] S.C. Tan, Y.M. Lai, and C.K. Tse, "A unified approach to the design of PWM based sliding mode voltage controller for basic DC-DC converters in continuous conduction mode", IEEE Transactions on Circuits and Systems I: Fundamental Theory and Applications, vol. 53, no. 8, pp. 1816-1827, Aug. 2006.

[8] L. Martinez-Salamero, J. Calvente, R. Giral, A. Poveda, and E. Fossas, "Analysis of a bidirectional coupled-inductor Cuk converter operating in sliding mode," IEEE Transactions on Circuits and Systems Part I: Fundamental Theory and Applications, vol. 45, no. 4, pp. 355-363, April 1998.

[9] S.C. Tan, Y.M. Lai, and C.K. Tse, "General design issues of sliding mode controllers in DC-DC converters", IEEE Transactions on Industrial Electronics, to appear. 